\title{
Creatina fosfocinasa MB como marcador diagnóstico de disfunción miocárdica en pacientes pediátricos con quemadura eléctrica
}

\author{
Mariana L. Miguel-Sardaneta ${ }^{*}$, Yaneth Martínez-Tovilla², Fernando D. Solís-Cordero ${ }^{3}$, \\ Mary S. Saavedra-Pacheco ${ }^{4}$ y Manuel Gil-Vargas ${ }^{3}$ \\ ${ }^{1}$ Servicio de Pediatría, Hospital General Zona Norte de Puebla, Servicios de Salud del Estado de Puebla; ${ }^{2}$ Unidad Pediátrica de Quemados (Anexo \\ a Hospital del Niño Poblano), Servicios de Salud del Estado de Puebla; ${ }^{3}$ Servicio de Cirugía Pediátrica, Hospital General de Puebla Eduardo \\ Vázquez Navarro, Servicios de Salud del Estado de Puebla; ${ }^{4}$ Facultad de Medicina, Benemérita Universidad Autónoma de Puebla; Puebla, México
}

\begin{abstract}
Resumen
Introducción: La disminución de la fracción de eyección ventricular izquierda (FEVI) como consecuencia de una disfunción ventricular se reporta dentro de las alteraciones cardiacas secundarias a una lesión eléctrica. Como complemento de la exploración física, la ecografía cardiaca enfocada (FoCUS) permite una evaluación rápida, no invasiva, que da información para contribuir a tomar mejores decisiones terapéuticas, sobre todo en pacientes en estado crítico. El objetivo de este estudio fue explorar la utilidad de la creatina fosfocinasa MB (CPK-MB) como marcador diagnóstico de disfunción miocárdica en pacientes de 6 a 18 años con quemadura eléctrica. Métodos: Durante el periodo de noviembre de 2018 a agosto de 2019 se llevó a cabo un estudio transversal analítico de 10 pacientes, de 6 a 18 años de edad, con quemadura eléctrica, en quienes se obtuvo el porcentaje de FEVI a través del protocolo FoCUS. Posteriormente, el porcentaje de FEVI se correlacionó con los valores de CPK-MB y el porcentaje de superficie corporal quemada (SCQ) en las primeras 24 horas después de la lesión. Resultados: Se encontraron 10 casos de quemadura eléctrica, ocho de sexo masculino y dos de sexo femenino, con una media de edad de 13 años. El 80\% de estos casos presentó disminución leve de la FEVI (45-59\%). Al realizar el protocolo FoCUS se reportó hipocinesia miocárdica en siete pacientes. Se encontró una correlación moderada entre la FEVI y los valores de CPK-MB ( $\rho=-0.671)$, aunque no se observó correlación entre la FEVI y la SCQ. Conclusiones: $L a$ ecografía cardiaca influye en la toma de decisiones y mejora el pronóstico de estos pacientes.
\end{abstract}

Palabras clave: Fracción de eyección ventricular izquierda (FEVI). Quemadura eléctrica. Ecografía cardiaca enfocada (FoCUS).

\section{Creatine phosphokinase MB levels as a diagnostic marker of myocardial dysfunction in pediatric patients with electrical burns}

\section{Abstract}

Background: The decrease of the left ventricular ejection fraction (LVEF) as consequence of a ventricular dysfunction is reported in cardiac alterations secondary to electrical injury. The focused cardiac ultrasound (FoCUS) helps to complete the clinical examination because it allows a faster non-invasive evaluation, and provides information that contributes to make

Correspondencia:

*Mariana L. Miguel-Sardaneta

E-mail: mmiguel.s@ hotmail.com
Disponible en internet: 13-11-2020 Bol Med Hosp Infant Mex. 2020;77(6):320-326

www.bmhim.com 1665-1146/@ 2020 Hospital Infantil de México Federico Gómez. Publicado por Permanyer. Este es un artículo open access bajo la licencia CC BY-NC-ND (http://creativecommons.org/licenses/by-nc-nd/4.0/). 
better therapeutic decisions, especially those for patients in critical condition. The objective of this study was to explore the utility of creatine phosphokinase MB (CPK-MB) as a diagnostic tool of myocardial dysfunction in patients from 6 to 18 years old with electrical burn. Methods: From November 2018 to August 2019, we conducted a transversal analytic study of 10 children with electric burn (6 to 18 years of age), in whom the percentage of LVEF was obtained through the FoCUS proto$\mathrm{col}$ in the first 24 hours after injury. Results: We found 10 cases of electrical burn injury, eight males and two females, with an average of 13 years of age. Eighty percent of these cases showed a slight decrease in LVEF (45-59\%). When performing the FoCUS protocol, myocardial hypokinesia was reported in seven patients. We observed a moderate correlation between LVEF and CPK-MB levels $(\rho=-0.671)$, and no correlation between LVEF and body surface area affected by the electrical burn. Conclusions: The cardiac ultrasound influences decision making to improve the prognosis of these patients.

Key words: Left ventricular ejection fraction (LVEF). Electrical burn. Focused cardiac ultrasound (FoCUS).

\section{Introducción}

Las quemaduras son lesiones causadas por la transferencia aguda de energía que genera desde zonas de hiperemia hasta una respuesta inflamatoria sistémica que busca detener y reparar dicho daño'.

El trauma eléctrico en general es resultado del contacto con cables eléctricamente activos o rayos. La corriente puede transmitirse a los tejidos profundos antes de que esta cause un daño significativo de la piel. Por ello, no es posible evaluar el grado de lesión interna basándose en la gravedad de la quemadura externa ${ }^{2}$.

La energía eléctrica se transforma en calor a medida que la corriente pasa a través de los tejidos del cuerpo con mala conducción. Las lesiones por quemaduras eléctricas son generadas por la colisión de los electrones al paso de la corriente eléctrica a través de un conductor y la liberación de energía térmica, lo que ocasiona daño a los componentes de la membrana celular y provoca la desnaturalización de las proteínas. Este daño conlleva la formación de poros y la subsecuente muerte celular. Este proceso, conocido como electroporación, altera el potencial y la función de la membrana celular ${ }^{3-5}$.

La necrosis celular ocurre después de 1 segundo de exposición a $69{ }^{\circ} \mathrm{C}$ o de 1 hora de exposición a $45^{\circ} \mathrm{C}$. La necrosis muscular profunda ocurre adyacente al hueso. Por tanto, el daño muscular ocasionado por la transformación de energía eléctrica en calor produce una necrosis progresiva debido a que la mayor parte de la corriente viaja a lo largo de las líneas de menos resistencia, particularmente de los vasos sanguíneos. Estos vasos lesionados experimentarán una oclusión posterior, ocasionando necrosis isquémica progresiva de los músculos que nutren, edema tisular con riesgo de desarrollar síndrome compartimental y rabdomiólisis. El grado de lesión muscular se relaciona con las concentraciones séricas de creatina fosfocinasa $(\mathrm{CPK})^{6}$.

La magnitud de la lesión depende de la resistencia del tejido (determinada por la estirpe, el tamaño celular, el grosor y el estado de hidratación del tejido), la intensidad de la corriente, el tiempo de exposición y la vía que recorre la corriente a través del cuerpo.

Las lesiones que involucran la caja torácica son las que causan mayor necrosis en el tejido cardiaco. El corazón es uno de los órganos más vulnerables a la corriente eléctrica. Aunque la fibrilación ventricular es la causa más común de muerte por esta lesión, puede condicionar alteraciones en el segmento ST y la onda $\mathrm{T}$, extrasístoles, taquicardias de origen supraventricular, fibrilación auricular, bloqueos auriculoventriculares, asistolia o rotura miocárdica, así como una disminución de la fracción de eyección ventricular izquierda (FEVI) como consecuencia de una disfunción ventricular ${ }^{7,8}$.

La literatura internacional reporta que aproximadamente el $31 \%$ de los pacientes con quemaduras por electricidad presentan anormalidades en la función del ventrículo izquierdo, aun cuando no exista compromiso hemodinámico ${ }^{9}$.

De manera sistemática, en toda quemadura se debe cuantificar la superficie corporal quemada (SCQ). Sin embargo, en el caso de una quemadura por electricidad, una lesión de superficie pequeña puede tener repercusiones graves. En pacientes quemados por electricidad de alto voltaje, las alteraciones funcionales y de estructuras profundas serían mayores que el daño cutáneo. Por lo tanto, la SCQ no es tan relevante en este tipo de quemaduras como lo suele ser en las quemaduras térmicas, por lo que no es un factor pronóstico de importancia. Asimismo, se deben cuantificar la CPK, la CPK-MB y las troponinas en la sangre, ya que el daño cardiaco lleva a la liberación de estas enzimas miocárdicas. La ecografía cardiaca enfocada (FoCUS) es el complemento de la exploración física: determina la afección cardiaca y no necesariamente requiere ser 
medida por un cardiólogo. Además, permite una evaluación rápida, no invasiva, y ayuda de forma temprana a delimitar lesiones, como crecimiento ventricular, derrames pericárdicos, gradientes de presión o crecimiento de cavidades, para obtener información que ayude a tomar las mejores decisiones terapéuticas, sobre todo en aquellos pacientes en estado crítico ${ }^{10}$.

Un gran número de niños con quemaduras eléctricas presentan alteraciones cardiacas asociadas, por lo que resulta de utilidad evaluar los cambios ecocardiográficos en la población pediátrica (escolares y adolescentes) en las primeras 24 horas después de la lesión. Sin importar la SCQ, estas alteraciones ecocardiográficas se traducen clínicamente como repercusiones hemodinámicas importantes que aumentan la morbilidad y la mortalidad de los pacientes.

El objetivo de este estudio fue explorar la utilidad de la CPK-MB como marcador diagnóstico de disfunción miocárdica en pacientes de 6 a 18 años con quemaduras eléctricas.

\section{Métodos}

Se realizó un estudio transversal analítico con un muestreo no probabilístico a conveniencia. Se evaluaron 10 pacientes, de 6 a 18 años de edad, con quemadura eléctrica, atendidos durante el periodo comprendido de noviembre de 2018 a agosto de 2019 en la unidad de quemados de los Servicios de Salud del Estado de Puebla. Se tomaron en cuenta los criterios de inclusión (todo paciente que arribara dentro de las primeras 24 horas después de una quemadura eléctrica sin manejo previo, pacientes que contaran con protocolo FoCUS y cuyos padres otorgaran el consentimiento para entrar al protocolo de estudio), los criterios de exclusión (pacientes con quemaduras por otra causa y aquellos sin expediente clínico completo) y los criterios de eliminación (pacientes que fallecieron durante las primeras 24 horas, aquellos sin ecocardiografía con protocolo FoCUS y los que fueran trasladados a otra unidad hospitalaria antes de la medición de la FEVI).

Se realizó un estudio ecocardiográfico a todo paciente escolar 0 adolescente que presentara una lesión por electricidad dentro de las primeras 24 horas de ocurrida esta y sin manejo hemodinámico previo.

La ecocardiografía se realizó con el protocolo FoCUS (ultrasonido SIEMENS pediátrico y transductor ultrasónico Modelo 4V1c, banda 1-4.5 MHz, modo 2D) para determinar el porcentaje de FEVI. Por ser un estudio breve y encauzado a establecer si existe 0 no un número limitado de diagnósticos críticos, este protocolo permite realizar la ecocardiografía al lado de la cama del paciente en tiempo real y ayuda a obtener información que contribuye a la toma de decisiones terapéuticas. En este estudio se analizan los puntos subepicárdico, eje largo (donde se mide la FEVI), eje corto y cuatro cámaras, lo que permite valorar algunos puntos, como la presencia de derrame pericárdico, función sistólica del ventrículo e hipertrofia ventricular, entre otros ${ }^{11}$.

Personal experto revisó los expedientes clínicos de cada paciente y se creó una base de datos en SPSS Statistics versión 25 para Windows, en la que se organizaron las variables categóricas y se les otorgó un valor. Las variables que se recabaron fueron las siguientes: FEVI (normal, 60-80\%; disminución leve, 45-59\%; disminución moderada, 35-44\%; disminución grave, < $30 \%$ ), edad, sexo, SCQ (de acuerdo con la escala de Lund y Browder ${ }^{12}, 1-100 \%$ ), voltaje (voltios implicados en el mecanismo de lesión²: alto, > $1001 \mathrm{~V}$; bajo, < $1000 \mathrm{~V}$ ), CPK-MB, alteraciones registradas en el electrocardiograma, cambios ecocardiográficos (alteraciones registradas durante la realización del protocolo FoCUS distintas a la disminución de la FEVI: ninguna, hipocinesia miocárdica, derrame pericárdico), complicaciones asociadas (traumatismos registrados que se hayan derivado del mecanismo de lesión principal: ninguno, esguince cervical, traumatismo craneoencefálico grave, amputación de mano, contusión torácica, síndrome compartimental, choque hipovolémico, choque cardiogénico, neumotórax) y puntos de contacto (sitios anatómicos que tuvieron contacto con la corriente eléctrica).

Se realizó estadística descriptiva utilizando medidas de tendencia central y dispersión como media y desviación estándar (DE). Para la correlación de las variables numéricas se realizó la correlación de Spearman.

\section{Resultados}

Se estudiaron 10 pacientes que cumplieron con los criterios de inclusión; el $80 \%$ eran de sexo masculino y la media de edad era de 13 años (DE: 3.4 años).

Con relación a la FEVI, el $20 \%$ de los pacientes no presentó alteraciones (FEVI 60-80\%) y el resto presentó disminución leve (FEVI 45-59\%).

El punto de contacto más frecuente fueron las extremidades superiores e inferiores, y en la mayoría de los pacientes se observó una SCQ del 5-20\% (Tabla 1). El $30 \%$ de los pacientes no presentaron complicaciones 
Tabla 1. Frecuencia de puntos de contacto y superficie corporal quemada

\begin{tabular}{|c|c|c|c|}
\hline \multicolumn{2}{|c|}{ Variables categóricas } & \multirow[t]{2}{*}{ n } & \multirow{2}{*}{$\begin{array}{l}\% \\
80\end{array}$} \\
\hline Sexo & Masculino & & \\
\hline & Femenino & 2 & 20 \\
\hline \multirow{8}{*}{$\begin{array}{l}\text { Punto de } \\
\text { contacto más } \\
\text { frecuente }\end{array}$} & Tórax & 1 & 10 \\
\hline & $\begin{array}{l}\text { Genitales, extremidades } \\
\text { superiores e inferiores }\end{array}$ & 1 & 10 \\
\hline & $\begin{array}{l}\text { Tórax, pelvis y extremidades } \\
\text { superiores }\end{array}$ & 1 & 10 \\
\hline & $\begin{array}{l}\text { Abdomen, extremidades } \\
\text { superiores e inferiores }\end{array}$ & 1 & 10 \\
\hline & $\begin{array}{l}\text { Tórax, abdomen, extremidades } \\
\text { superiores e inferiores }\end{array}$ & 1 & 10 \\
\hline & $\begin{array}{l}\text { Extremidades superiores e } \\
\text { inferiores }\end{array}$ & 2 & 20 \\
\hline & Pelvis y extremidades inferiores & 1 & 10 \\
\hline & Extremidades superiores & 2 & 20 \\
\hline \multirow{3}{*}{$\begin{array}{l}\text { Superficie } \\
\text { corporal } \\
\text { quemada }\end{array}$} & $5-20 \%$ & 7 & 70 \\
\hline & $23-25 \%$ & 2 & 20 \\
\hline & $43 \%$ & 1 & 10 \\
\hline
\end{tabular}

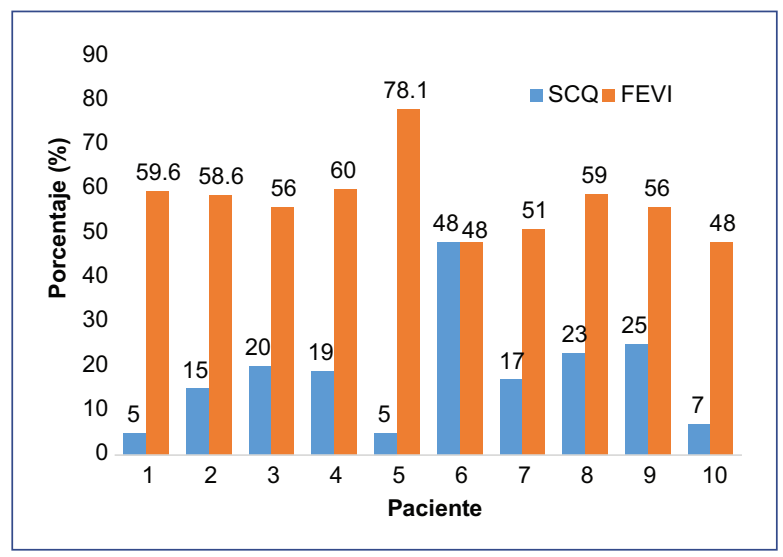

Figura 1. Superficie corporal quemada (SCQ) y fracción de eyección ventricular izquierda (FEVI) de cada paciente.

asociadas con la lesión eléctrica; el esguince cervical fue la complicación más frecuente.

De los 10 pacientes, ocho presentaron disfunción ventricular izquierda leve de acuerdo con su FEVI (45-59.9\%). De estos ocho, solo cinco presentaron una SCQ del $5-20 \%$ (Figura 1). Sin embargo, todos los pacientes presentaron elevación de la CPK-MB (Figuras 2 y 3 ).

Al realizar la ecografía cardiaca con el protocolo FoCUS se reportó hipocinesia miocárdica en siete pacientes y derrame pericárdico en uno. La media de los valores de CPK-MB fue de $1532 \mathrm{UI} / \mathrm{L}$, y la de SCQ fue del $18 \%$.

Al realizar la correlación de Spearman entre la FEVI y la SCQ se obtuvo un valor $\rho$ de -0.428 (intervalo de confianza del 95\% [IC95\%]: -0.83 a 0.28) (Figura 4), y entre la FEVI y los valores de CPK-MB se obtuvo un valor de $\rho$ de -0.671 (IC95\%: -0.91 a -0.07) (Tabla 2).

\section{Discusión}

La ecocardiografía cambia la impresión clínica y el plan terapéutico en casi la mitad de los pacientes a quienes se realiza. Por ello, el protocolo FoCUS adquiere un gran valor como herramienta de diagnóstico y monitoreo hemodinámico en cuidados intensivos pediátricos, lo que enfatiza la importancia de un programa de entrenamiento riguroso $0^{13}$.

Este estudio se diseñó para investigar los cambios en la FEVI mediante la realización de ecocardiografía con el protocolo FoCUS y para correlacionar la disminución de la FEVI con los valores de la CPK-MB y el porcentaje de SCQ en pacientes que sobrevivieron a una lesión eléctrica (Figura 5).

Los resultados muestran que existe una correlación negativa entre la disminución de la FEVI y las concentraciones séricas de CPK-MB $(-0.671, p<0.05)$, lo que indica que, a menor $\mathrm{FEVI}$, mayor es la concentración de CPK-MB (Figura 2). Con respecto a la FEVI y la $S C Q$, no se observó una correlación estadísticamente significativa y se detectó una correlación negativa media (Figura 4).

Kim, et al. ${ }^{7}$ mostraron que las alteraciones en la cinética miocárdica después de una lesión eléctrica de alto voltaje no se asocian con una disminución de la contractilidad regional o global del ventrículo izquierdo, y por lo tanto no reportaron repercusión en la FEVI.

Aunque algunos estudios han demostrado que el daño miocárdico puede ocurrir independientemente del voltaje (alto o bajo) ${ }^{14,15}$, la evaluación de la lesión cardiaca según los cambios del electrocardiograma 0 unos resultados poco confiables de CPK-MB parece ser inadecuada para determinar la presencia o la extensión del daño en el miocardio. Sin embargo, como se aprecia en este estudio, existe una correlación considerable, lo que debe incrementar la 


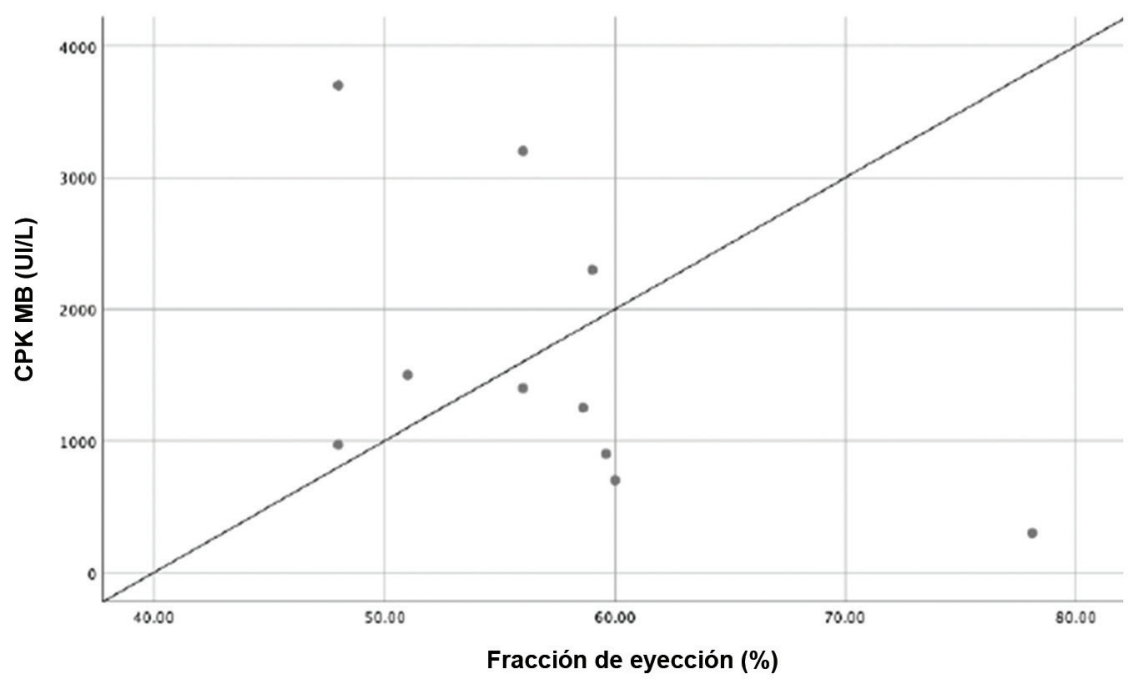

Figura 2. Gráfica de dispersión entre la concentración de creatina-fosfocinasa MB (CPK-MB) y la fracción de eyección del ventrículo izquierdo.

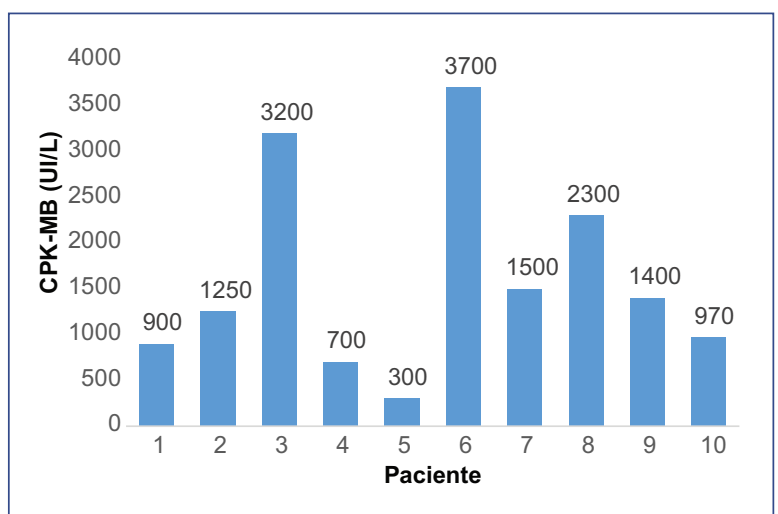

Figura 3. Valores de creatina-fosfocinasa MB en los pacientes con quemaduras eléctricas.

sospecha de alteración en la cinética del miocardio y, por lo tanto, llevar a la realización de ecografía cardiaca con protocolo FoCUS.

Por otra parte, estudios previos han mostrado conflictos en los resultados con respecto al daño miocárdico asociado con lesiones eléctricas ${ }^{16-19}$. Las razones principales para estas diferencias pueden estar relacionadas con tamaños de muestra pequeños y datos insuficientes para soportar el diagnóstico de daño al tejido miocárdico. Esto se observó en el presente estudio, cuya principal limitación fue el número de pacientes evaluados y la realización del protocolo FoCUS por un solo observador.
Tabla 2. Correlaciones entre la fracción de eyección ventricular izquierda y las variables determinadas en los pacientes con quemaduras eléctricas

\begin{tabular}{|l|c|c|c|}
\hline Marcador & $\boldsymbol{\rho}^{*}$ & $\mathbf{p}$ & IC95\% \\
\hline CPK-MB & -0.671 & 0.038 & -0.91 a -0.07 \\
\hline SCO & -0.428 & 0.217 & -0.83 a 0.28 \\
\hline
\end{tabular}

CPK-MB: creatina-fosfocinasa MB; IC95\%: intervalo de confianza del $95 \%$; SCQ: superficie corporal quemada. *Correlación de Spearman.

Lino, et al. ${ }^{17}$ notaron daño miocárdico en la mayoría de los pacientes con lesión eléctrica de alto voltaje, pero la ecocardiografía convencional no fue lo suficientemente sensible para detectar daños sutiles en el miocardio en comparación con otros métodos de imagen, como las imágenes de radionúclidos, que detectaron daños significativos.

Si bien se evaluaron las alteraciones cardiacas en las primeras 24 horas del daño, es indispensable el seguimiento ecocardiográfico después de la lesión eléctrica, ya que, de acuerdo con Guinard, et al. ${ }^{20}$, aquellos pacientes afectados por alto voltaje pueden persistir con anormalidades cardiacas a largo plazo, lo que justifica su seguimiento con ecocardiogramas seriados. Este es un motivo importante para la capacitación del personal médico en la realización del protocolo FoCUS. 


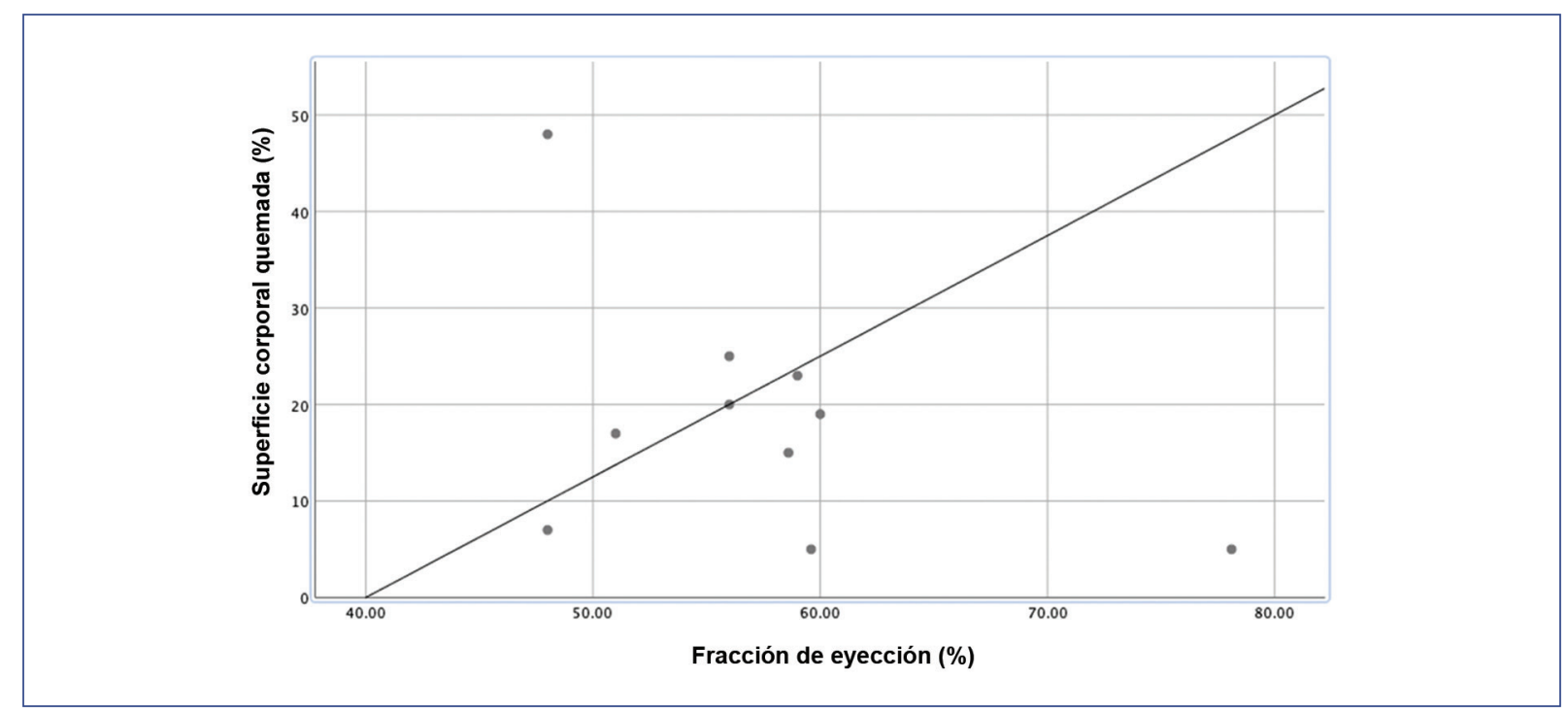

Figura 4. Dispersión entre la superficie corporal quemada y la fracción de eyección ventricular izquierda en los pacientes con quemaduras eléctricas.

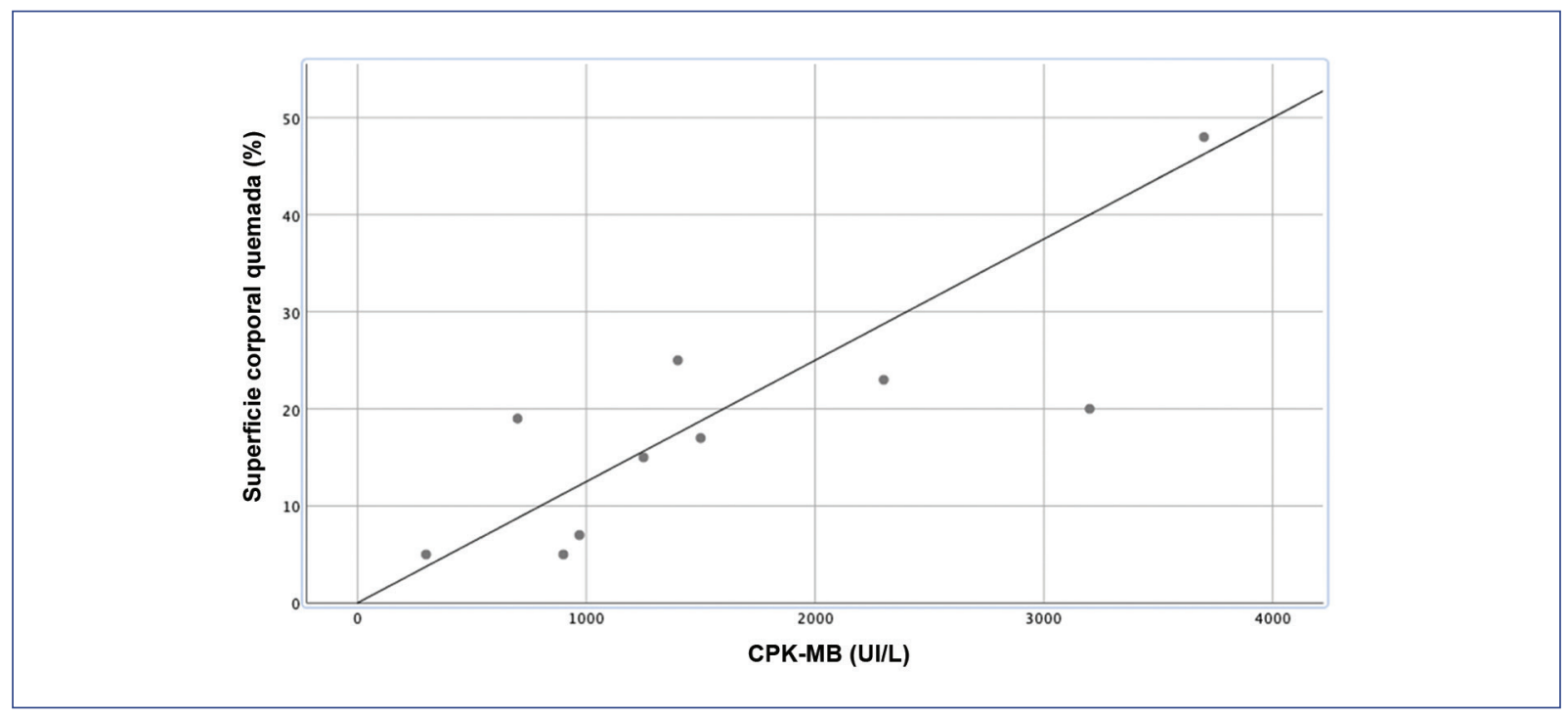

Figura 5. Dispersión entre la superficie corporal quemada y la concentración de creatina-fosfocinasa MB (CPK-MB) en los pacientes con quemadura eléctricas.

En este estudio se demostró evidencia estadísticamente significativa de la correlación entre la disminución de la FEVI y los valores de CPK-MB en pacientes con quemadura eléctrica. Esta correlación podría contribuir a la realización de la ecografía cardiaca e influir en la toma de decisiones para mejorar el pronóstico de estos pacientes. En cambio, no se encontró diferencia estadísticamente significativa para la SCQ, ya que, en caso de quemadura eléctrica, una lesión pequeña puede tener repercusiones graves.

En virtud de que la disminución de la FEVI ocurre en pacientes con quemaduras por electricidad, es indispensable que el personal médico de los centros especializados posea las habilidades y destrezas para la realización del protocolo FoCUS lo antes posible, con la finalidad de realizar las intervenciones 
oportunas para el soporte hemodinámico y prever complicaciones.

Existe evidencia de que las anormalidades en la función cardiaca pueden persistir, por lo que se justifica el seguimiento a largo plazo con ecocardiografías seriadas en los pacientes que las presenten.

\section{Responsabilidades éticas}

Protección de personas y animales. Los autores declaran que para esta investigación no se han realizado experimentos en seres humanos ni en animales.

Confidencialidad de los datos. Los autores declaran que han seguido los protocolos de su centro de trabajo sobre la publicación de datos de pacientes.

Derecho a la privacidad y consentimiento informado. Los autores han obtenido el consentimiento informado de los pacientes y/o sujetos referidos en el artículo. Este documento obra en poder del autor de correspondencia.

\section{Conflicto de intereses}

Los autores declaran no tener ningún conflicto de intereses.

\section{Financiamiento}

Ninguno.

\section{Bibliografía}

1. Moctezuma L, Páez I, Jiménez S, Miguel K, Foncerrada G, Sánchez A Epidemiología de las quemaduras en México. Rev Esp Med Quir. 2015;20:78-82.
2. Jain S, Bandi V. Electrical and lightning injuries. Crit Care Clin. 1999;15:319-31.

3. Glatstein M, Ayalon I, Miller E, Scolnik D. Pediatric electrical burn injuries experience of a large tertiary care hospital and a review of electrical injury. Pediatr Emerg Care. 2013;29:737-40.

4. Waldmann V, Narayanan K, Combes N, Marijon E. Electrical injury. BMJ. 2017;357:j1418.

5. Lee RC. Injury by electrical forces: pathophysiology, manifestations, and therapy. Curr Probl Surg. 1997;34:677-764.

6. McGill MP, Kamp TJ, Rahko PS. High-voltage injury resulting in permanent right heart dysfunction. Chest. 1999;115:586-7.

7. Kim S, Cho G, Kim M, Park W, Kim J, Lim H, et al. Alterations in left ventricular function assessed by two-dimensional speckle tracking echocardiography and the clinical utility of cardiac troponin I in survivors of high-voltage electrical injury. Crit Care Med. 2009;37:1282-7.

8. Leyva JC, Carvajal-Flechas F. Lesiones eléctricas. Univ Med Bogota. 2015;56:63-74.

9. Pastor-Torres L, Sáenz de Tejeda-Morales A, Seco-Vasco J, Sáinz-Hidalgo I, Vázquez-García R. [Ventricular functional changes following an episode of accidental electrocution]. Rev Esp Cardiol. 1995; 48:66-9.

10. Spence K, Kimura B, Korcarz C, Pellikka P, Rahko P, Siegel R. Focused cardiac ultrasound: recommendations from the American Society of Echocardiography. J Am Soc Echocardiogr. 2013;26:567-81.

11. Neskovic AN, Edvardsen T, Galderisi M, Garbi M, Gullace G, Jurcut R, et al. Focus cardiac ultrasound: the European Association of Cardiovascular Imaging viewpoint. Eur Hear J Cardiovasc Imaging. 2014;15: 956-60.

12. Núñez LV, García LL, Jiménez GS, Reyes SJ. Guía de práctica clínica. Diagnóstico y tratamiento de quemaduras en pediatría en el primer nivel de atención. México: Secretaría de Salud; 2008. p. 6.

13. Rato J, Camilo C, Boto L, Rios J, Abecasis F, Vieira M. The impact of focused cardiac ultrasound performed by pediatric intensivists. Pediatr Emerg Care. 2019. doi: 10.1097/PEC.0000000000001885. Online ahead of print.

14. Homma S, Gillam L, Weyman A. Echocardiographic observations in survivors of acute electrical injury. Chest. 1990;97:103-5.

15. Arnoldo B, Klein M, Gibran N. Practice guidelines for the managment of electrical injuries. J Burn Care Res. 2006;27:439-47.

16. Chandra N, Siu C, Munster A. Clinical predictor of myocardial damage after high voltage electrical injury. Crit Care Med. 1990;18:293-7.

17. Lino $H$, Chikamori T, Hatano $T$, Morishima $T$, Hida S, Yanagisawa $H$, et al. Hightension electrical injury to the heart as assessed by radionuclide imaging. Ann Nucl Med. 2002;16:557-61.

18. McBride J, Labrosse K, McCoy H, Ahrenholz DH, Solem LD, Goldenberg IF. Is serum creatine kinase-MB in electrically injured patients predictive of myocardial injury. JAMA. 1986;255:764-8.

19. Martínez-Téllez D, Martínez-Tovilla Y García-Villaseñor A. Disfunción ventricular izquierda en pacientes pediátricos con quemaduras por electricidad. Rev Mex Pediatr. 2017;84:10-4.

20. Guinard J, Chiolero R, Buchser E, Delaloye-Bischof A, Payot M, Grbic A, et al. Myocardial injury after electrical burns: short and long term study. Scand J Plast Reconstr Surg Hand Surg. 1987;21:301-2. 